\title{
NASA Propulsion Sub-system Concept Studies and Risk Reduction Activities for Resource Prospector Lander
}

\author{
Huu P. Trinh \\ NASA Marshall Space Flight Center \\ Huntsville, Alabama
}

\begin{abstract}
NASA's exploration roadmap is focused on developing technologies and performing precursor missions to advance the state of the art for eventual human missions to Mars. One of the key components of this roadmap is various robotic missions to Near-Earth Objects, the Moon, and Mars to fill in some of the strategic knowledge gaps. The Resource Prospector (RP) project is one of these robotic precursor activities in the roadmap. RP is a multi-center and multiinstitution project to investigate the polar regions of the Moon in search of volatiles. The mission is rated Class D and is approximately 10 days, assuming a five day direct Earth to Moon transfer.

Because of the mission cost constraint, a trade study of the propulsion concepts was conducted with a focus on available low-cost hardware for reducing cost in development, while technical risk, system mass, and technology advancement requirements were also taken into consideration. The propulsion system for the lander is composed of a braking stage providing a high thrust to match the lander's velocity with the lunar surface and a lander stage performing the final lunar descent. For the braking stage, liquid oxygen (LOX) and liquid methane (LCH4) propulsion systems, derived from the Morpheus experimental lander, and storable bi-propellant systems, including the 4th stage Peacekeeper (PK) propulsion components and Space Shuttle orbital maneuvering engine (OME), and a solid motor were considered for the study. For the lander stage, the trade study included miniaturized Divert Attitude Control System (DACS) thrusters (Missile Defense Agency (MDA) heritage), their enhanced thruster versions, ISE-100 and ISE-5, and commercial-off-the-shelf (COTS) hardware.

The lowest cost configuration of using the solid motor and the PK components while meeting the requirements was selected. The reference concept of the lander is shown in Figure 1. In the current reference configuration, the solid stage is the primary provider of delta- $\mathrm{V}$. It will generate 15,000-lbf of thrust with a single burn of $\sim 80$ 's seconds. The lander stage is a bipropellant, pressure-regulated, pulsing liquid propulsion system to perform all other functions.
\end{abstract}




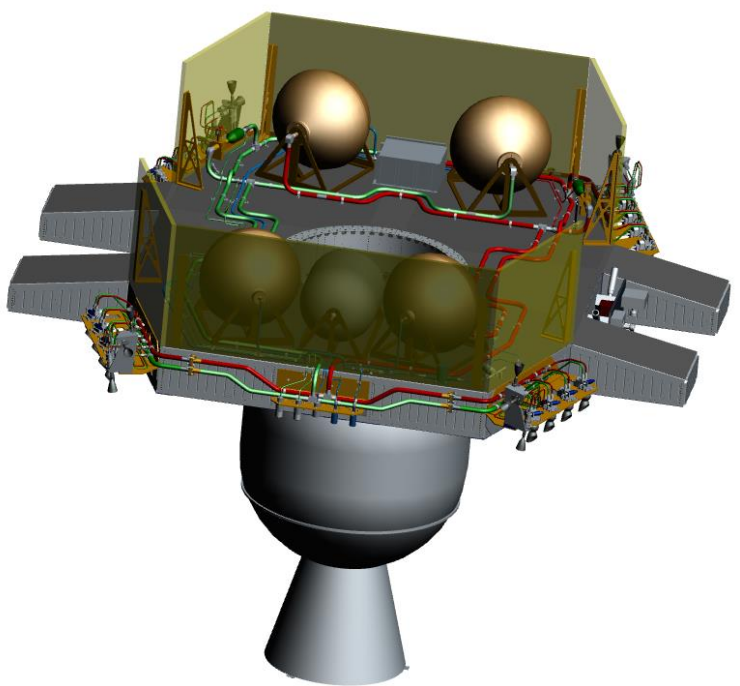

Figure 1: Government version of RP lander. SRM for braking stage and a bi-prop system for lander stage.

The flow schematic and design layout of the liquid propulsion for this lander are presented in Figures 2 and 3, respectively. Hypergolic propellants monomethylhydrazine (MMH) and nitrogen tetroxide (NTO) will be used to fuel sixteen 70-lbf class descent thrusters and twelve 5-lbf attitude control thrusters. These thrusters are grouped into four thruster modules that are located on corners of the lander. All thrusters will operate with pulsing modes for precision and soft landing. The descent thrusters will provide main thrust for trajectory correction maneuvers and terminal descent, while the ACS thrusters will perform pitch, roll, yaw, and nutation damping.

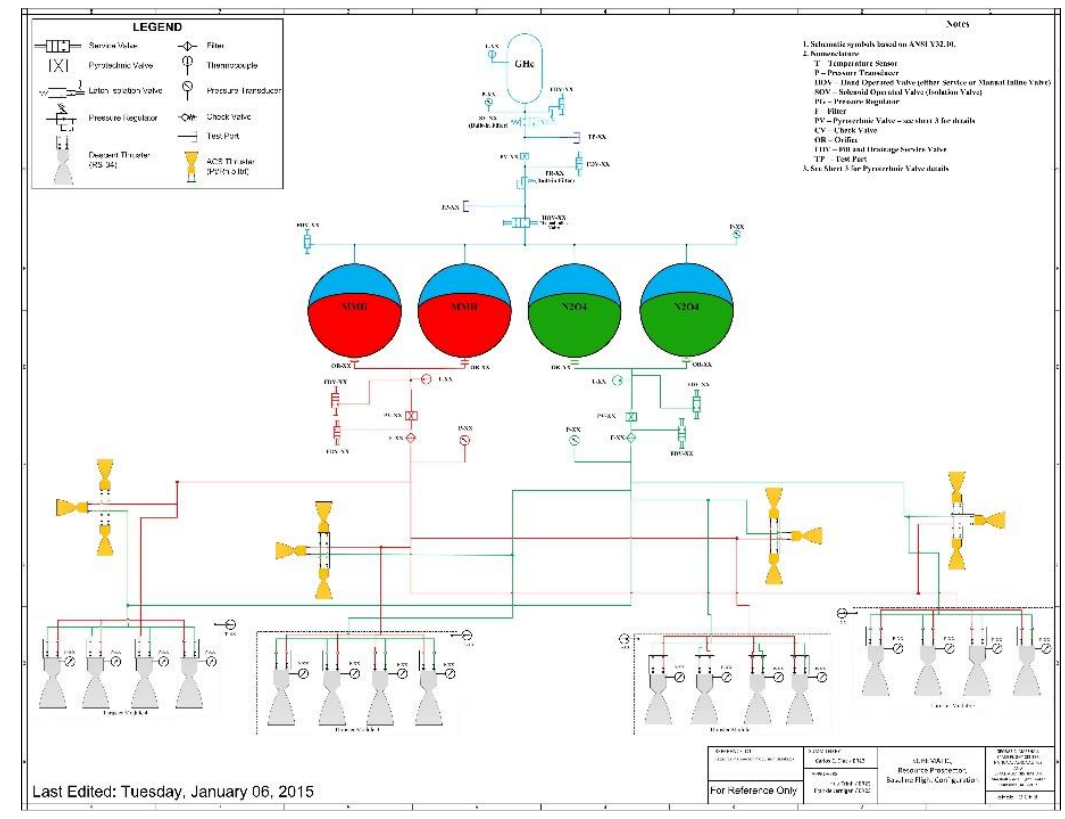

Figure 2: Flow schematic of liquid propulsion for lander stage 


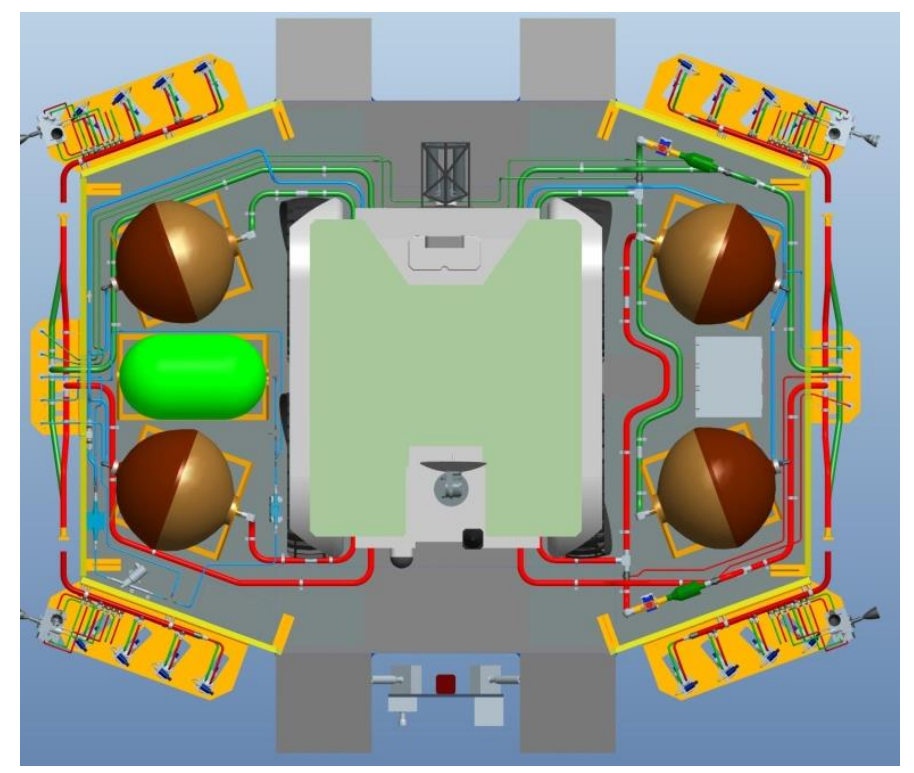

Figure 3: Layout design of liquid propulsion system

With respect to the propellant storage and distribution system, four metal diaphragm tanks, two connected-in-parallel tanks per propellant component, will be used along with a highpressure composite overwrapped pressure vessel (COPV) for the helium pressurant gas. The metal diaphragm tanks offer the advantage of active propellant control to eliminate technical issues, such as propellant slosh and gaseous pressurant entrainment to the engines, while gaining high propellant expulsion efficiency ( 98\%). Using government available PK propulsion components along with some commercial off-the-shelf (COTS) hardware would significantly reduce the cost of the spacecraft while maintaining the technical and schedule risk at a minimal level.

Moving forward in maturing the liquid propulsion concept, a series of risk reductions including the system design, the propulsion system cold flow test, and thruster hot-fire tests were also conducted. For the cold flow test, a simulated propulsion system based on the drawings of the early flight design was built to evaluate the feasibility of using available PK and COTS propulsion components in the lander application. This buildup also serves as a mockup for demonstrating the integration of a propulsion system to a flight-like lander structure. The propulsion cold flow test provided data to characterize the steady state flow and pressure conditions as well as transient behavior. The testing included a focused parametric study (variations in operating conditions, simulated variations in metal diaphragm pressure drops, etc.) on steady state operation, slump and waterhammer effects due to the combination of opening and closing of the thruster valves, and system priming (initial system activation). Additional repeated tests were performed to assess the flight hardware integrity ( $4 \mathrm{x}$ life cycles) including its ability to satisfy the safety margins. The integration of the propulsion system to the lander structure can be depicted in Figure 4. Dimensions of the lander structure are roughly 10-ft by $10-\mathrm{ft}$. The flight drawings at the time were utilized to build-up the propulsion cold flow test system. Though the flight drawings were used, deviations occurred based on cost constraints, schedule constraints including component availability, producibility, and test considerations. 


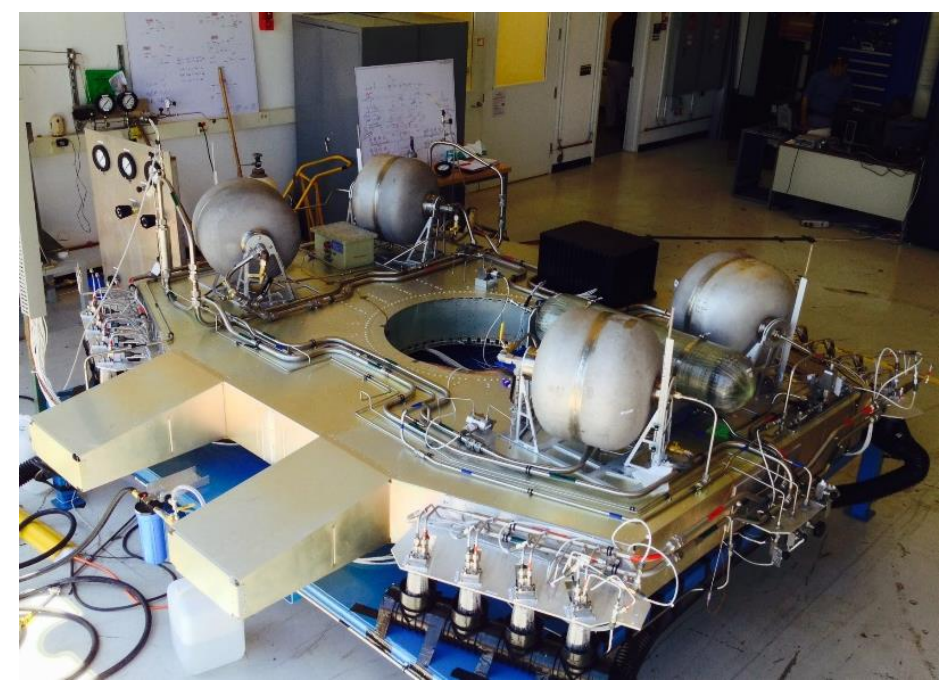

Figure 4: Integration of propulsion cold flow test system to lander structure

For the descent thrusters, RS-34 70-lbf thrusters used on the 4th stage PK propulsion are selected for the lander stage. However, the usage of such thrusters for the RP mission is slightly outside of the qualified regime of the RS-34. Moreover, the aging of the hardware is also a concern for the project. To reduce the risk of using the thrusters for the mission, a hot-fire test program on the thruster was conducted in vacuum conditions at NASA White Sand Test Facility (WSTF) in New Mexico. Figure 5 shows a top view of the thrusters with the instrumentation potted to the surface of the chamber and nozzle. Some thermocouples are not visible in this view, but are attached to the underside of the nozzle and the valve body. Also, not shown in the picture are 6 uni-axial accelerometers used for combustion stability assessments. The thruster test setup in the vacuum chamber is shown in figure 6 . A total of 88 hot-fire tests were successfully carried on two RS-34 thrusters.

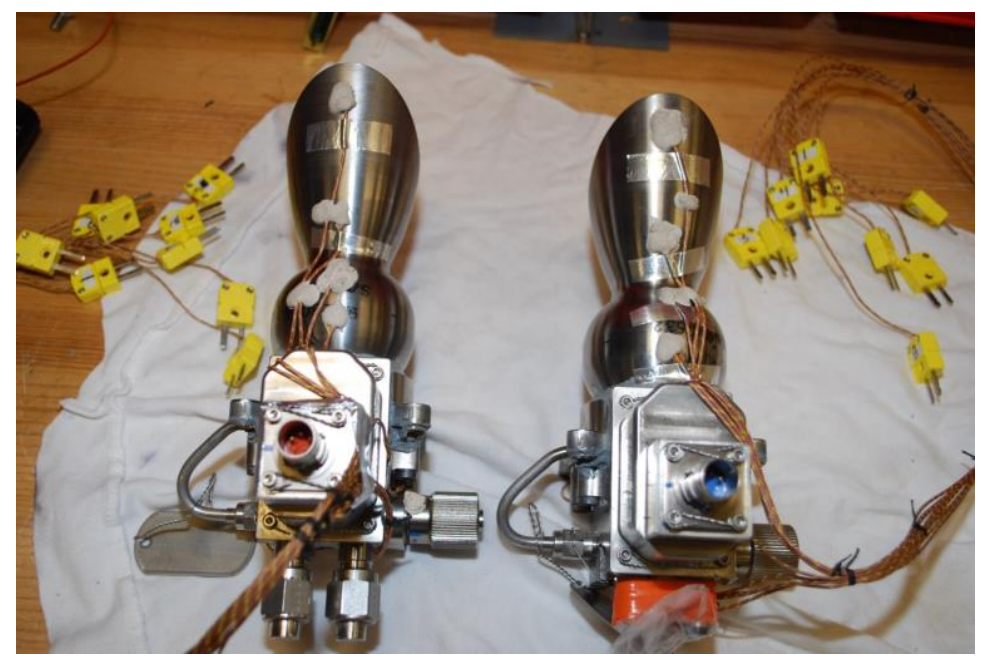

Figure 5: Instrumentation on the RS-34 thrusters for hot-fire test 


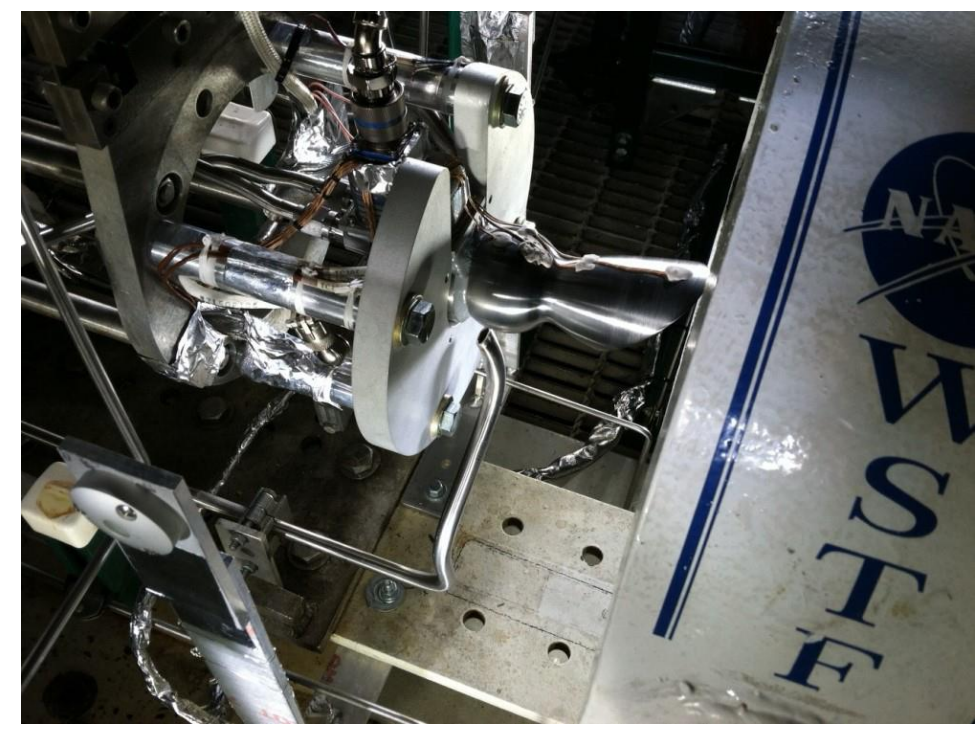

Figure 6: RS-34 thruster test setup in a vacuum chamber at WSTF

This paper will provide an overview of the propulsion concept studies and following-on risk reduction activities. The selected propulsion concept will also be discussed and results of the risk reduction efforts will be highlighted on the report. 